\title{
Infrared and speckle image processing applied to heterogeneous thermomechanical behaviour of polymers
}

\author{
B. Wattrisse, J.-M. Muracciole, A. Chrysochoos
}

Université Montpellier II, Laboratoire de Mécanique et de Génie Civil, Equipe Thermomécanique, Place E. Bataillon, C.C. 081, 34095 Montpellier Cedex 5, E-mail : wattriss@lmgc.univ-montp2.fr

\begin{abstract}
This paper presents an application of quantitative infrared thermography in the field of solid mechanics. The processing of thermal images of a sample, recorded during tensile tests performed on a semi-crystalline polymer under its glass transition temperature, gives the distribution of the heat sources responsible for the temperature variations. These energetic data are associated to other mechanical information (strain, strain-rate, stress distribution) by means of a speckle image processing technique.

These tests show the early and progressive development of the mechanical, and the resulting calorimetric manifestations of necking.
\end{abstract}

\section{Introduction :}

The theory of Irreversible Processes Thermodynamics sets a convenient framework for the description of the materials behaviour. For solid material, the temperature and the strain tensor are often chosen as the two first state variables of thermomechanical models.

On the one hand, infrared thermography is a very reliable way to estimate quantitatively the distribution of temperature on the surface of thin and flat samples. These data may be used to derive the heat source pattern inducing the temperature variations: local energy measurements are of a great practical interest to propose particular forms of constitutive equations and to identify the material parameters of the models.

The interpretation of these calorimetric data often requires the simultaneous knowledge of other mechanical variables such as stress and strain tensors. Classically, these local variables are supposed to be homogeneous and they are measured at a macroscopic scale. To be consistent with the energetic measurements, the stress and strain variables should also be determined locally. Digital image correlation methods were developed to get such local kinematic measurements (strain, strain-rate tensors). The determination of the stress pattern, without making strong assumptions on the material behaviour, is more delicate. We have developped a method, based on the integration of the equilibrium equations, to compute some components of the stress tensor.

In this paper, we deal with the case of a semi-crystalline thermoplastic polymer submitted to a quasi-static, monotonic tensile test. This polymer shows a strong softening at the yield stress, and it is subjected to local necking. The calorimetric results obtained on this material are compared qualitatively and quantitatively with kinematic measurements corresponding to similar testing conditions.

\section{Experimental set-up :}

\subsection{Thermal measurements}

The experimental set-up involves a $100 \mathrm{kN}$ tension-compression, servo-mechanic testing machine. An infrared camera is placed in the front of the testing machine to record temperature charts of the sample surface during the test. The heat equation (Eq. 1) can relate, under certain conditions, the temperature variations to the volumic heat sources 
induced by the straining. In the framework of our experiments this equation can be simplified as [1] :

$$
\rho C\left(\frac{\partial \theta}{\partial \mathrm{t}}+\frac{\theta}{\tau_{\mathrm{th}}}\right)-k \Delta \theta=w_{\mathrm{ch}}^{\prime}
$$

where $\rho, C$, and $\tau_{\text {th }}$ are the mass density, the heat capacity, and a time constant characterising heat losses by convection and radiation, respectively. The variable $\theta$ stands for the temperature variation between the current and reference states. The volume heat source $w_{\mathrm{ch}}^{\prime}$ can then be derived from an estimate of the left hand member of Eq. (1). It is a partial derivative operator applied to the temperature variations : $\partial / \partial \mathrm{t}$ and $\Delta$ symbolise the partial time derivative and the Laplacian operator, respectively.

In order to estimate precisely the second member of Eq. (1), the noisy thermal data have to be filtered before any differentiation. The estimate of the derivatives was performed by using a local least-square fitting of data. The thermal data are approximated by a parabolic function, corresponding to a locally constant conduction heat losses, on a chosen neighbourhood of each pixel of the thermal image. The dimensions of the approximation zone affect the smoothing of the data, and thus they influence the accuracy of the filtering. Validity checks of the method were realised (see section 2.3), and constant approximation zones of $15 \times 15$ square pixels were shown to give a good compromise between speed and accuracy.

We have chosen to implement a centred processing of the temperature charts. So the algorithms require data on each side of the current pixel. To calculate the heat sources on each side of the image, a data extension (regularity: $\mathrm{C}_{2}$ ) was made before each computational step. The temperature field is approximated using the data available on one side of the boundary, and the temperature of the points located on the other side is given by the approximation function.

\subsection{Kinematic measurements}

The digital images are recorded during the test by a C.C.D. camera, set in front of the sample. The lens axis of the camera is kept fixed with reference to the frame of the testing machine, perpendicular to the surface of the sample. The image processing is realised after the test itself, and can be split up into two steps. First, the displacement field is estimated. The displacement of each material particle is decomposed into 2 in-plane components and 1 out-of-plane component. The two first can be computed by a correlation technique. Between two images $I_{1}$ and $I_{2}$, separated by a small strain increment, the displacement (in pixels) of a given point $M$, located at pixel $(i, j)$ in the initial image, is given by the couple $(k, l)$ maximising the intercorrelation function $\bar{\varphi}$ defined by [2] :

$$
\overline{\bar{\varphi}}(k, l)=\frac{\sum_{i=-C Z / 2}^{C Z / 2} \sum_{j=-C Z / 2}^{C Z / 2} I_{1}(i, j) \cdot l_{2}(i+k, j+l)}{\sqrt{\sum_{i=-C Z}^{2}, \frac{R Z}{2} \sum_{j=-C Z / 2}^{C Z / 2} I_{1}^{2}(i, j)} \cdot \sqrt{\sum_{i=-C Z / 2}^{C Z / 2} \sum_{j=-C Z / 2}^{C Z / 2} I_{2}^{2}(i+k, j+l)}}
$$

where :

- $C Z$ is the number of pixels defining the correlation zone (i.e. $M$ 's neighbourhood corresponding to a local optical signature of point $M$ )

- $R Z$ is the number of pixels defining the research zone

To reach a subpixel resolution, a local quadratic interpolation is performed in the vicinity of the discrete maximum of $\bar{\varphi}[3]$. 
The third displacement component can be reached by assuming material incompressibility. The strain (or the strain-rate) field is then derived from the displacements by space (and time) differentiation. To reach a sufficient accuracy on the kinematic measurements, the displacement field have to be filtered too. Here we have also adopted a local approximation smoothing technique to process the kinematic data: the approximation function is linear, and it is applied to the field of each component of the displacement.

Note that, at the present time, the two cameras are not set simultaneously in front of the sample, and the energetic and kinematic data are not recorded on the same sample.

\subsection{Validation}

The performances of these image processing were tested both on analytic and experimental cases corresponding to homogeneous or heterogeneous heat sources distribution (energetic approach), or to homogeneous or heterogeneous straining (kinematic approach).

The validity check of the out-of-plane displacement computation was performed by comparing the thickness evolution of a steel sample after a tensile test, given by a three-axis measurement machine with the one given by the computation [4].

The influence of all the computation parameters on the energetic and kinematic measurements was tested. Using standard parameters, the accuracy on the temperature is around $10^{-2}{ }^{\circ} \mathrm{C}$, and on the displacement calculation, it is about $5.10^{-2}$ pixel and $5.10^{-4}$ on the strain measurement [4].

\section{Results :}

The kinematic and thermal response of a semi-crystalline thermoplastic was studied, at room temperature, in the case of tensile testing. The material response was investigated for uniaxial, monotone loading performed on ISO R527 samples. Heat source and the strain-rate fields were found to be rather homogeneous along the sample width while they strongly changed along the longitudinal axis. This result led us to propose a one-dimensional representation of these data. Therefore, results are graphically illustrated with time evolution of axial distribution of the heat sources $\left(w_{\mathrm{ch}}^{\prime}(x, 0, t)\right)$, and with the time evolution of the axial distribution of the tension component of the Lagrangian strain rate tensor $\left(\dot{E}_{x x}(x, 0, t)\right)$.

Figure 1 shows the case of a test performed on the thermoplastic previously mentioned. In the foreground, the tension curve represented by the nominal stress against conventional strain $\left(\sigma_{\mathrm{N}}=F / S_{0}, \varepsilon_{\mathrm{C}}=\left(I-I_{0}\right) / I_{0}\right)$ is plotted. It gives a useful link between local measurements and the current macroscopic mechanical state. The parameter $F$ stands for the applied force, $S_{0}$ is the initial cross section, $I$ and $I_{0}$ are the current and initial gauge length, respectively.

Remember that the results presented in Figure 1 were not obtained simultaneously on the same sample, so that the observation zone slightly differed. Nevertheless, we can observe the strong similarities between these two independent experimental measurements : a localised necking develops in the lower end of the sample, and propagates at constant velocity along the test section of the sample. The image processing evidenced that the material was not strained outside the necking zone : in these regions no storage or release of heat was observed. During its propagation, the necking zone is the only part of the sample to be actually strained. In Figure 1.a., we observe that a heat sources heterogeneity appears as soon as the applied force passes through its maximum. During the subsequent softening of the specimen, this heterogeneity concentrate progressively in the lower end of the sample. The necking zones starts its longitudinal propagation as soon as the applied force has reached the plateau. This progressive development can also be spotted on the kinematic 
response : the loci where the strain-rate is the most important correspond to the loci where the intensity of the heat sources is the highest.

As soon as a kinematic or an energetic heterogeneity appears, it is legitimate to wonder about the homogeneity of the stress distribution within the sample. In a first step, we consider that the tensile stress $\sigma(M, t)$ at each point of the sample axis is given by :

$$
\sigma(M, t)=\sigma_{N}(t) e^{\varepsilon(M, t)}
$$

where $\varepsilon(M, t)$ is the longitudinal Hencky strain measured at point $M(x, 0)$ and at time $t$.

In the case of plane stress, using the tensile stress distribution, and integrating the equilibrium equations gives the two other components of the stress tensor, without any other assumption on the material behaviour. More details on the stress tensor construction are available in [5].

Figure 2 shows the time-evolution of the spatial distribution of the computed tensile stress $\sigma(x, 0, t)$. By construction, the spatial variations of the stress field are the same as the strain variations, and the stress pattern naturally concentrates during necking. Furthermore, we can observe that, during all the test duration, the strained zone continues to harden.

The mechanical behaviour of the material can be illustrated by plotting the local correspondence between stress and strain for each point of the sample. Figure 3 gives these responses $(\sigma(M, t), \varepsilon(M, t))$ for several points located on the sample axis. We first notice that the material responses are not identical : the point located in the middle of the necking zone (A) is always hardening, whereas the points located away from this zone seem to soften for a time then harden again ( $D, C$ and $D)$, or to always soften (E). Furthermore, we can observe that when the points harden, the evolution of the hardening modulus is very similar from one point to another.

\section{Concluding comments}

We developed two imaging techniques providing whole-field calorimetric and kinematic data during mechanical tests. These two techniques are deeply complementary : for a part, heat sources are related to the intrinsic dissipation which is linked to the power of internal forces (and so to the strain-rate). These thermomechanical data give useful information to observe and analyse material behaviour.

In particular, tests realised on polymers have shown narrow zones of strain localisation where strain-rates, heat sources and thus temperature variations are very important.

First, knowing that polymers are thermo-sensitive, the experimental findings confirm, as it was expected, the great importance of developing behavioural models in a framework able to take into account the thermomechanical coupling mechanisms.

Second, the precocity of localisation inception lead us to consider the sample as a structure and fully justify the employment of imaging techniques to get whole-field measurements.

Third, for dynamic loading or strongly localised deformation mode, it is necessary to combine thermal and kinematic data to reach calorimetric information. When convective terms are no longer negligible, a proper computation of the particular time derivative $(\mathrm{d} / \mathrm{dt}=\partial / \partial \mathrm{t}+\underline{v} \cdot \underline{\mathrm{grad}})$ requires either the knowledge of the temperature spatial distribution and the velocity field. And finally, when mechanical fields are no longer homogeneous, this simultaneous measurement enables to realise a local energy balance. 


\section{References}

[1] CHRYSOCHOOS, A. , "Analyse du comportement des matériaux par thermographie infrarouge", Colloque PhotoMécanique 95, pp 201-211, 1995

[2] BAILEY, H.H., BLACKWELL, F.W., LOWERY, C.L., RATKOVIC, J.A., "Image correlation : Part I. Simulation and analysis, report prepared for United States Air Force Project RAND, R2057/1-PR, 1976

[3] OULAMARA, A., TRIBILLON, G., DUVERNOY, J., "Subpixel speckle displacement measurement using a digital processing technique", J. Mod. Opt., 35, 1988

[4] WATTRISSE, B., CHRYSOCHOOS, A., MURACCIOLE, J.-M., NEMOZ-GAILLARD, M., " Analysis of strain localisation during tensile tests by Digital Image Correlation", Exp. Mech., in revision

[5] WATTRISSE, B., CHRYSOCHOOS, A., MURACCIOLE, J.-M., NEMOZ-GAILLARD, M., " Kinematic manifestations of localisation phenomena in steels by Digital Image Correlation", Eur. J. of Mech. - Part A/Solids, accepted

\section{Figures}

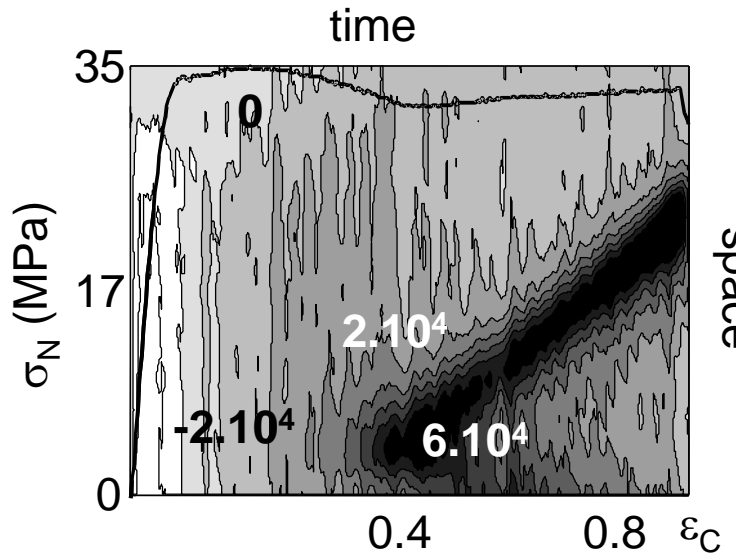

(a)

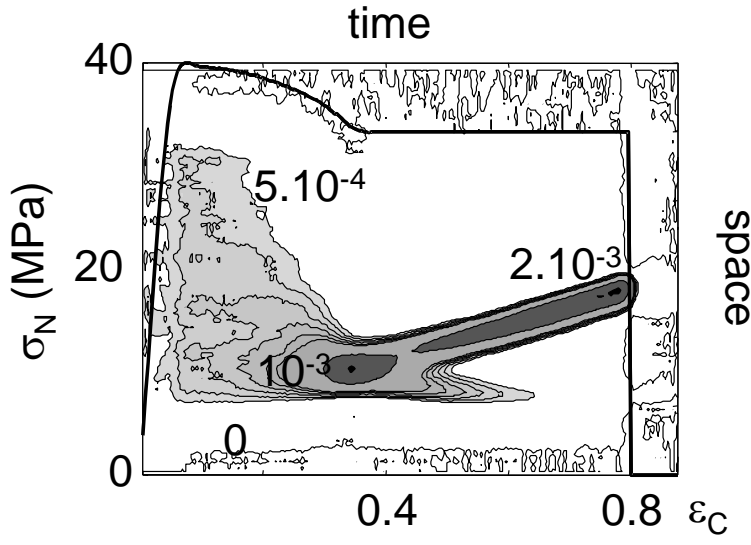

(b)

Figure 1 : Space-time calorimetric and kinematic responses of a semi-crystalline thermoplastic sample submitted to a monotone, velocity controlled, tensile test, performed at room temperature - (a) evolution of the heat sources distribution $w_{c h}^{\prime}(x, 0, t)$ in $W \cdot m^{-3}$ for an imposed velocity $v_{\mathrm{CH}}=0.04 \mathrm{~mm} \cdot \mathrm{s}^{-1}-$ (b) evolution of the strain rate distribution $\dot{E}_{\mathrm{xx}}(x, 0, t)$ in $\mathrm{s}^{-1}$ for an imposed velocity $v_{\mathrm{CH}}=0.05 \mathrm{~mm} \cdot \mathrm{s}^{-1}$ 


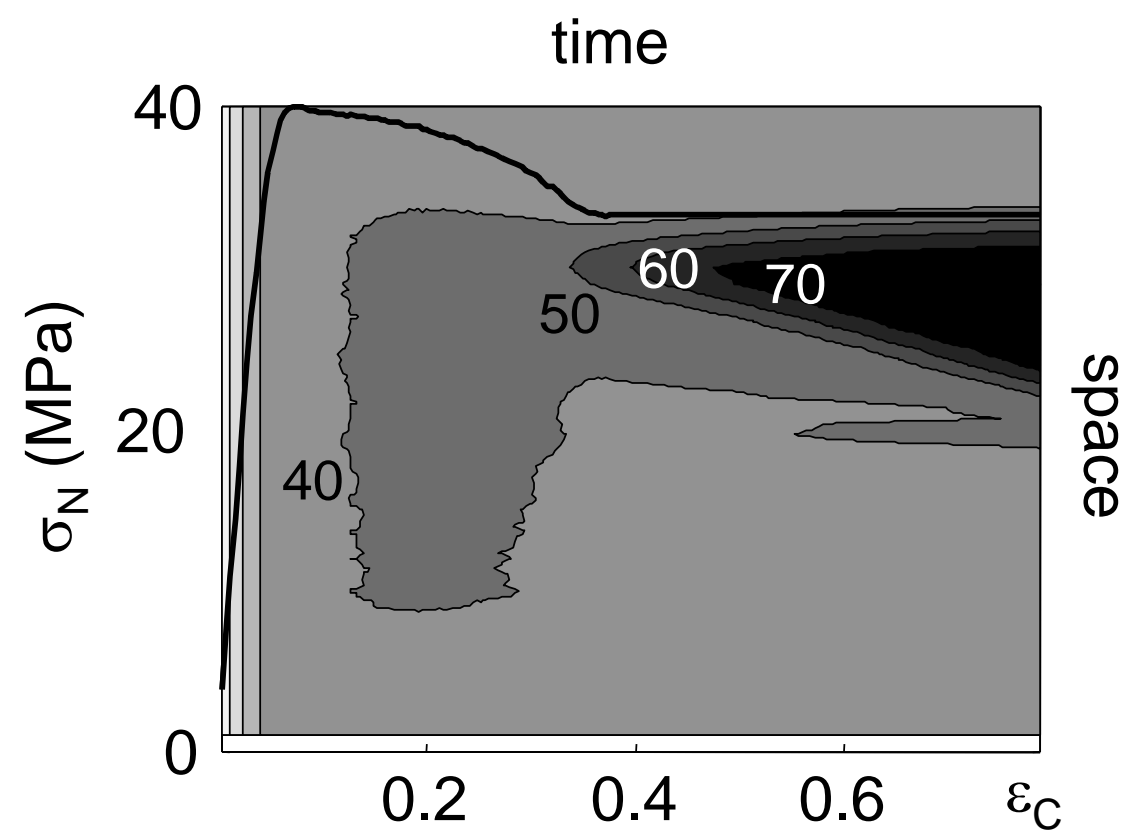

Figure 2: Evolution of the tensile stress distribution $\sigma(x, 0, t)$ in $\mathrm{MPa}$ for an imposed velocity $v_{\mathrm{CH}}=0.05 \mathrm{~mm} \cdot \mathrm{s}^{-1}$ on a semi-crystalline thermoplastic sample submitted to a monotone, velocity controlled, tensile test, performed at room temperature.

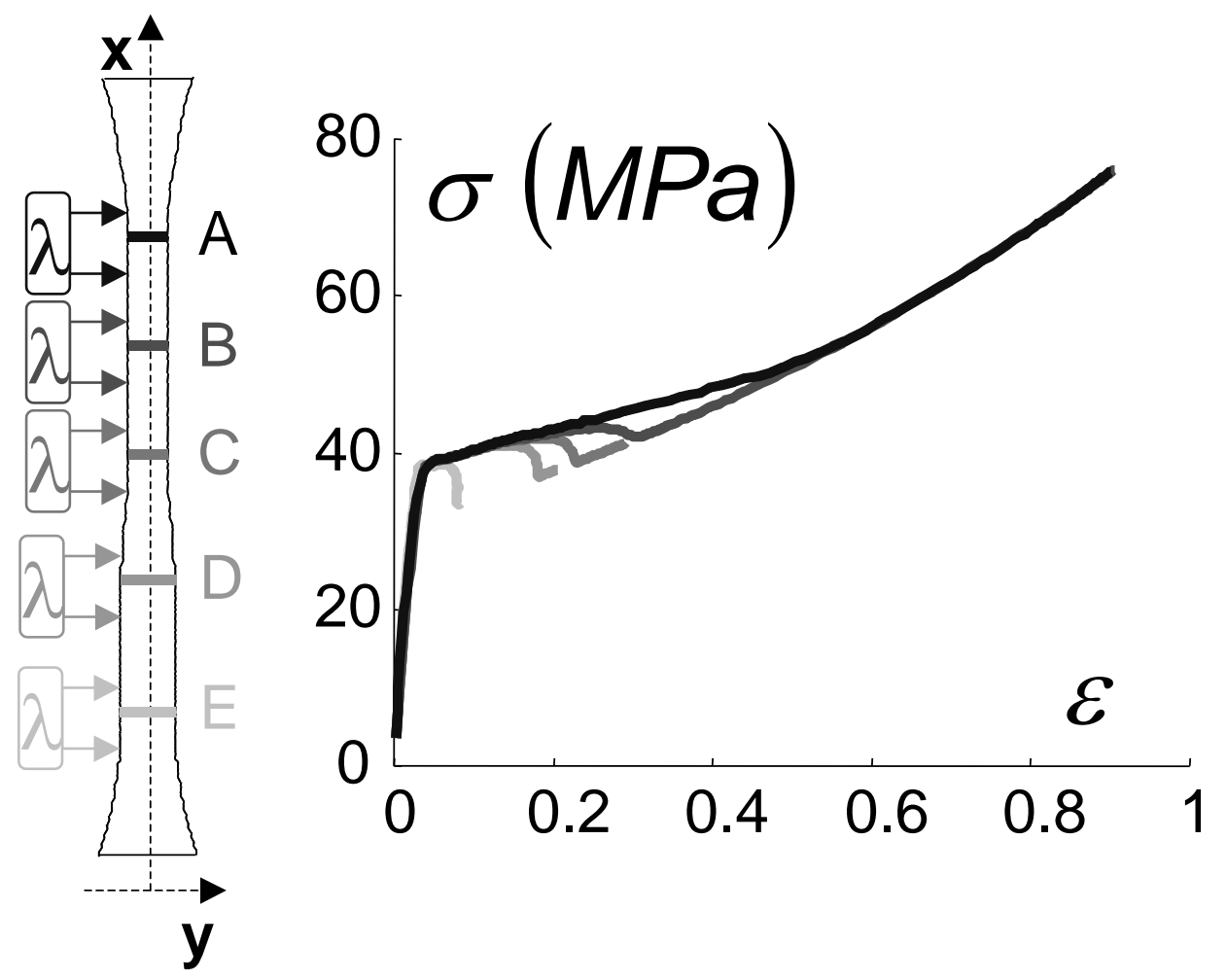

Figure 3 : Local mechanical responses $(\sigma(M, t), \varepsilon(M, t))$ for points located on the sample axis, during a tensile test performed on a thermoplastic polymer 